\title{
Shear bond strength of orthodontic brackets bonded using halogen light and light-emitting diode at different debond times
}

\section{Rebeca Di Nicoló(a) \\ Maria Amelia Maximo de Araujo(b) \\ Levy Anderson César Alves ${ }^{(c)}$ \\ Rodrigo Othavio de Assunção \\ e Souza ${ }^{(\mathrm{d})}$ \\ Daniel Maranha da Rocha ${ }^{(d)}$}

\section{(a) Associate Professor; (c) Graduate Student - Department of Orthodontics and Pediatric Dentistry, Faculdade de Odontologia de São José dos Campos, Unesp - Univ Estadual Paulista, São José dos Campos, SP, Brazil. \\ (b) Associate Professor, Department of Restorative Dentistry, Faculdade de Odontologia de São José dos Campos, Unesp - Univ Estadual Paulista, São José dos Campos, SP, Brazil. \\ (d) MSc, Graduate Student, Department of Prosthesis, Faculdade de Odontologia de São José dos Campos, Unesp - Univ Estadual Paulista, São José dos Campos, SP, Brazil.}

\begin{abstract}
The aim of this in vitro study was to compare the photoactivation effects of QTH (Quartz-Tungsten-Halogen) and LED (Light-Emitting Diode) on the SBS (Shear Bond Strength) of orthodontic brackets at different debond times. Seventy-two bovine lower incisors were randomly divided into two groups according to the photoactivation system used (QTH or LED). The enamel surfaces were conditioned with Transbond self-etching primer, and APC (Adhesive Pre-Coated) brackets were used in all specimens. Group I was cured with QTH for $20 \mathrm{~s}$ and Group II with LED for $10 \mathrm{~s}$. Both groups were subdivided according to the different experimental times after bonding (immediately, $24 \mathrm{~h}$ and 7 days). The specimens were tested for SBS and the enamel surfaces were analyzed according to the Adhesive Remnant Index (ARI). The statistical analysis included the Tukey's test to evaluate the main effects of photoactivation and debond time on SBS. The Chi-square test was used to compare the ARI values found for each group, and no statistically significant difference was observed. The debond time of 7 days for QTH photoactivation showed statistically greater values of SBS when compared to the immediate and $24 \mathrm{~h}$ periods. There was no statistically significant difference between the QTH and LED groups immediately and after the $24 \mathrm{~h}$ period. In conclusion, bonding orthodontic brackets with LED photoactivation for $10 \mathrm{~s}$ is suggested because it requires a reduced clinical chair time.
\end{abstract}

Descriptors: Orthodontic brackets; Curing lights, dental; Dental debonding; Dental etching.

\section{Introduction}

Since 1955, when Buonocore ${ }^{1}$ introduced the acid-etching bonding technique, the concept of bonding resin-based materials to enamel has favored the development of its application in preventive and restorative procedures in dentistry, including the bonding of orthodontic brackets. ${ }^{2}$ Therefore, several materials have been developed in order to achieve an adequate clinical SBS (Shear Bond Strength) in bonding procedures, and also to prevent permanent enamel injury after debonding.

Orthodontists generally use the conventional technique of acid-etching to adhere brackets to enamel tissue. More recently, different types of adhesives have been made available for bracket bonding, combining 
the functions of primer and adhesive in one clinical step, with no need of etching and rising. The combination of conditioning and priming into a single step reduces the bonding time and increases the cost-effectiveness of the procedure for the clinician, and consequently for the patient. ${ }^{3-8}$

A variety of curing lights are available to photoactivate dental materials. However, the most commonly used method is the one that uses QTH (QuartzTungsten-Halogen) light. QTH-based lights degrade over time, and the power output can drop to levels which might not be sufficient to perform an adequate photoactivation of the material. Portable radiometers can easily measure the power density of most LCUs (Light-Curing Units), but most dentists are unaware of their LCU's power output until the light fails to operate. ${ }^{8}$ These lights generate high heat, which degrades the bulb's components over time, and are also very sensitive to shock and vibration. ${ }^{9}$

In 1995, Mills ${ }^{10}$ proposed a solid-state LED (Light-Emitting Diode) technology to photoactivate dental materials. Its main advantage is a minimal generation of heat. Reduced temperatures lead to a lifetime of over 10,000 hours, with no significant degradation in light output. LED units generate high-energy photons in the blue region $(470 \mathrm{~nm})$ of the visible light spectrum, ideally matched to the absorption wavelength of the camphoroquinone. Some units are cordless, are reliable in their power and spectral output, do not need a cooling fan, operate silently and are more resistant to shock and vibration. ${ }^{9,11,12}$

Some factors such as the intensity of the irradiation, wavelength, time of exposure and distance between the source and the specimen may affect the photoactivation process in composite resins. Increasing the time of exposure with standard QTH light directly increases the cure of composite resins, although in a nonlinear relationship. The higher the duration of the exposure, the greater the photoactivation.

LED and QTH units have shown higher mean values of SBS when the cure time was increased $(10,20$ or $40 \mathrm{~s})$. However, only a small increase in SBS values occurred after an exposure time of more than 40 s. $^{13}$ Although the period of 24 hours does not correspond to clinical orthodontic practice, in which archwire is usually placed after bracket bonding, most studies evaluating the SBS of orthodontic brackets have reported this period as a suggested time and have used it to compare their results to those of other studies. ${ }^{3,7,14}$ The aim of the present study was to assess the effect of LED photoactivation on the SBS of orthodontic brackets at different debond times when compared to that of a traditional QTH system.

\section{Material and Methods}

A total of seventy-two freshly extracted bovine incisors, without any visible enamel defects were cleaned and disinfected with $0.5 \%$ chloramine-T solution. The crown specimens were mounted in epoxy cylinders, with the labial surface exposed and parallel to the basis of the cylinder, and randomly assigned to one of the six experimental groups.

The samples were prepared according to the following steps before bonding:

1. Sanded with n. 200,400, and 600-grit silicon carbide paper (3M, São Paulo, SP, Brazil) in order to produce a flat surface without exposing dentin;

2. polished for $10 \mathrm{~s}$ with a rubber prophylaxis cup and fluoride-free pumice and water;

3. rinsed for $15 \mathrm{~s}$ with distilled water;

4. air dried for $5 \mathrm{~s}$;

5. enamel conditioned with Transbond Plus SEP (Self-Etching Primer - 3M Unitek, Miami, FL, USA) for $5 \mathrm{~s}$ according to the manufacturer's instructions;

6. gently air dried.

APC (Adhesive Pre-Coated), stainless steel, maxillary incisor brackets of the Gemini Series (3M Unitek, Monrovia, CA, USA) were placed on the bonding surface. Excess adhesive was removed with a sharp explorer, and the samples were light-cured following the conditions assigned to each group.

Group I was cured with QTH for 20 s, 10 s on the mesial surface and $10 \mathrm{~s}$ on the distal surface of the bracket, using an Optilux VCL 403 (Demetron Research Corp., Danbury, CT, USA) and light intensity of $550 \mathrm{~mW} / \mathrm{cm}^{2}$. Group II was cured with LED for $10 \mathrm{~s}, 5 \mathrm{~s}$ on the mesial surface and $5 \mathrm{~s}$ on the distal surface of the bracket, using a LED-Ra- 
dii (SDI Limited, Victoria, Australia) and light intensity of $1000 \mathrm{~mW} / \mathrm{cm}^{2}$. The Light intensity of both systems was measured using a radiometer (Curing Radiometer Model 100 Demetron Research Corp., Danbury, CT, USA) before bonding. Both groups were subdivided into three groups $(\mathrm{n}=12)$ according to the debond times: immediately (a), 24 hours (b) and 7 days (c).

A mounting jig was used to align the labial surface of the tooth perpendicularly to the bottom of the base. Each tooth was oriented with the testing device as a guide such that its labial surface was parallel to the force during the shear strength test. A steel rod with one flattened end was attached to the cross-head of a universal testing machine EMIC DL2000 (EMIC Equip. Sist. Ensaio Ltda., São José dos Pinhais, PR, Brazil). An occlusogingival load was applied to the bracket, producing a shearing force at the bracket-tooth interface. A computer connected to the testing machine recorded the results of each test. SBS values were measured at a crosshead speed of $1 \mathrm{~mm} / \mathrm{min}$ and a $50-\mathrm{kg}$ load cell. The force was directly recorded in $\mathrm{N}$ (Newtons) and converted into $\mathrm{MPa}$ (megapascal).

All groups debonded after $24 \mathrm{~h}$ and 7 days were stored in distilled water at $37^{\circ} \mathrm{C}$, until the SBS test was performed. After debonding, teeth and brackets were examined by a blinded investigator under a light microscope $(20 \mathrm{X})$ to determine the mode of bond failure based on an assessment of the ARI (Adhesive Remnant Index). ${ }^{15}$ ARI ranged from 0 to 3 , following the scores defined as follows:

- $0=$ no adhesive left on the tooth;

- $1=$ less than half of the adhesive left on the tooth;

- 2 = more than half of the adhesive left on the tooth;

- 3 = adhesive totally left on the tooth with a distinct impression of the bracket mesh.

Descriptive statistics, including the mean and standard deviation, were calculated for each of the groups tested. Tukey's test was used to classify the groups in a homogeneous subset, and the ARI scores were compared by using the Chi-square test. Significance for all statistical tests was pre-determined at $p<.05$.

\section{Results}

The descriptive statistics on shear bond strength for the experimental groups are presented in Table 1.

Tukey's Test $(5 \%)$ was performed in order to compare the six experimental conditions and the results are shown in Table 2.

The distribution of ARI frequencies are presented in Table 3. The Chi-square test revealed no statistically significant difference among the groups: $p$-value $=.098>.05$.

\begin{tabular}{|c|c|c|c|c|c|c|}
\hline & & & & & & \\
\hline ble 1 - Descriptive statistics & & Group I (QHT) & & & Group II (LED) & \\
\hline standard deviation & a & $b$ & c & a & $b$ & c \\
\hline & $17.62 \pm 4.54$ & $17.95 \pm 2.84$ & $24.47 \pm 5.12$ & $16.11 \pm 3.82$ & $15.89 \pm 5.49$ & $20.06 \pm 5.01$ \\
\hline
\end{tabular}

Table 2 - Statistical comparison between all QTH and LED Groups by means of the Tukey's Test (5\%).

\begin{tabular}{c|c|c|c|c}
\hline Polymerization & Time & Mean & \multicolumn{2}{|c}{$\begin{array}{c}\text { Homogeneous } \\
\text { Groups }\end{array}$} \\
\hline Group I (QTH) & a (immediately) & 17.622 & & B \\
\hline Group I (QTH) & b (24 h) & 17.948 & & B \\
\hline Group I (QTH) & c (7 days) & 24.469 & A & \\
\hline Group II (LED) & a (immediately) & 16.108 & & B \\
\hline Group II (LED) & b (24 h) & 15.886 & & B \\
\hline Group II (LED) & c (7 days) & 20.058 & AB & \\
\hline
\end{tabular}

Table 3 - ARI (Adhesive Remnant Index) for the QTH and LED Groups.

\begin{tabular}{c|c|c|c|c}
\hline Groups / Index & 0 & 1 & 2 & 3 \\
\hline Group I a & 1 & 8 & 0 & 3 \\
\hline Group I b & 0 & 7 & 0 & 5 \\
\hline Group I c & 0 & 7 & 0 & 5 \\
\hline Group II a & 1 & 7 & 0 & 4 \\
\hline Group II b & 0 & 4 & 0 & 8 \\
\hline Group II c & 0 & 4 & 0 & 8 \\
\hline
\end{tabular}




\section{Discussion}

During function, orthodontic brackets are subjected to either shear, tensile or torsion forces, or even a combination of these factors. According to Reynolds $^{16}$ (1975), clinically adequate bond strengths to enamel for metal orthodontic brackets should range between 8 and $9 \mathrm{MPa}$, values that are sufficient to withstand normal orthodontic forces. In our study, the bond strength values observed were $20.01 \pm 5.24$ MPa for Group I and 17.35 $\pm 5.07 \mathrm{MPa}$ for Group II, therefore greater than those clinically accepted (Table 1).

As regards the different types of enamel conditioning - using the traditional system or a Self-Etching Primer (SEP) - SBS values may vary according to different studies. However, similar mean values of SBS, with no statistical difference between the methods mentioned previously, were observed in some studies..$^{17,18}$ In contrast, several authors ${ }^{3,6,14,19,20}$ have reported greater values of SBS for orthodontic brackets with phosphoric acid conditioning as compared to conditioning with a SEP. On the other hand, examination of the enamel-adhesive interface by scanning electron microscopy has revealed different patterns. Enamel conditioning with Transbond SEP has resulted in a more conservative enamel surface, comparable to that produced by traditional phosphoric acid conditioning. ${ }^{21-22}$ However, the SEP system may be considered for clinical use because its use may entail a decrease in undesirable enamel effects during bonding procedures, and also because it reduces clinical chair time. In addition, it is a less sensitive technique, which can be justified by its one-step procedure.

The use of APC brackets in the present study showed some advantages, such as standard quantity of adhesive, easy removal of excesses, better asepsis and reduction of occasional loss of material. Although the related literature has revealed greater mean values of SBS when using traditional orthodontic brackets, ${ }^{19,23,24}$ APC brackets were used instead because they involve the use of a less sensitive technique.

With regard to relationship between debonding time and SBS values, several studies have used a $24 \mathrm{~h}$ period after the bonding process. . $^{3,5,14,17,19,20,25-}$
${ }^{27}$ However, previous research has shown that orthodontic adhesives demonstrate greater SBS values after longer debonding periods of time. Nonetheless, the clinical practice of orthodontics requires that archwires be installed immediately after the bonding procedures.

Hence, many studies have been performed to evaluate the correlation between adhesive bond strength and post-bonding time. Hajrassie, $\mathrm{Khier}^{28}$ (2007) concluded that SBS values using orthodontic adhesives to metallic brackets increase with the debond time. Those authors ${ }^{28}$ evaluated periods of $10 \mathrm{~min}, 24 \mathrm{~h}, 7$ days, 1 week and 4 weeks after bonding, but no statistically significant difference was reported for both the in vivo and in vitro data. The results also indicated that, in vivo, the mean value (5.61 MPa) was significantly lower than in vitro $(13.98 \mathrm{MPa})$, thus contradicting the hypothesis about the similarity between the shear bond strengths values obtained in vivo and in vitro. The authors ${ }^{28}$ concluded that initial archwires could be placed 10 minutes after bonding.

The present study evaluated three different debond times. The results demonstrated that there were slight differences in debond time, and that the greatest values were observed in subgroup c ( 7 days), regardless of the type of photoactivation used (Table 1). The results also showed that the mean values were superior to $8 \mathrm{MPa}$. It should be borne in mind, however, that in vitro results are generally $40 \%$ higher than the results observed in vivo. ${ }^{28}$

With regard to the type of photoactivation, QTH units are traditionally preferred for bonding metallic orthodontic brackets, and have been used in most studies. $3,5,6,14,17,18,20-22,24$ Light-curing units (LCUs) must be able to bring enough energy to achieve the wavelength of the resin's photoinitiator to photoactivate. This physical-chemical process can be affected by light intensity, the material's composition and time of exposure. An effective photoactivation sometimes may be difficult to be achieved due to the depth of the prepared cavities, and also due to the thickness of the material, which sometimes exceeds $2 \mathrm{~mm} .{ }^{29}$ These aspects, however, are not present in the orthodontic clinic, since the resin adhesive thickness used for bonding brackets is smaller than 
$2 \mathrm{~mm}$, and because of the easier access to the material, in that the distance between the active point of the LCU and the bracket basis is small.

The Tukey's Test $(5 \%)$ indicated that two out of six experimental groups had the same performance (Table 2). From these findings it can be inferred that:

1. Group Ic differed from both Groups, Ia and Ib;

2. Group IIc did not differ from Groups IIa and IIb;

3. the four conditions established for subgroups a and $\mathrm{b}$ presented similar mean values, with no statistical difference.

The condition stated for Group IIc led to a performance which proved intermediate between the other groups, but statistically significant differences were observed only when considering Group Ic.

Based on the results obtained, it can be stated that $10 \mathrm{~s}$ of LED photoactivation provides enough adhesion for orthodontic accessories. Moreover, literature reports that SBS might be reduced when using shorter times of photoactivation $(<10 s){ }^{8,26,27}$

It is important to point out that clinicians should be aware of which photoinitiator is present in the orthodontic adhesive when using LED units due to its specific blue light range $(470 \mathrm{~nm})$, and also because other photoinitiators than camphoroquinone

\section{References}

1. Buonocore MG. A simple method of increasing the adhesion of acrylic filling materials to enamel surfaces. J Dent Res. 1955 Feb;34(6):849-53.

2. Newman GV. Epoxy adhesives for orthodontic attachments: progress report. Am J Orthod. 1965 Dec;51(12):901-12.

3. Buyukyilmaz T, Usumez S, Karaman AI. Effect of self-etching primers on bond strength - are they reliable? Angle Orthod. 2003 Feb;73(1):64-70.

4. Bishara SE, Oonsombat C, Ajlouni R, Laffoon JF. Comparison of the shear bond strength of 2 self-etch primer/ adhesive systems. Am J Orthod Dentofacial Orthop. 2004 Mar;125(3):348-50.

5. Rajagopal R, Padmanabhan S, Gnanamani J. A comparison of shear bond strength and debonding characteristics of conventional, moisture-insensitive, and self-etching primers in vitro. Angle Orthod. 2004 Apr;74(2):264-8.

6. Bishara SE, Ostby AW, Ajlouni R, Laffoon JF, Warren JJ. Early shear bond strength of a one-step self-adhesive on orthodontic brackets. Angle Orthod. 2006 Jul;76(4):689-93. (e.g. propanodiona) may not photoactivate adequately.

The ARI frequencies (Table 3) and Chi-square test did not reveal statistically significant differences between Groups I and II ( $p$-value $=0.098>.05$ ), which is supported by the literature. ${ }^{20,24,26}$ These findings probably occurred due to the use of APC brackets in all groups. Therefore, the same amount and the same type of adhesive were used as standard conditions for all the experiments.

\section{Conclusions}

- There were no statistically significant differences between the SBS values produced with the use of QTH and LED photoactivation immediately and $24 \mathrm{~h}$ after bonding.

- Bonding orthodontic brackets for $10 \mathrm{~s}$ using LED is suggested because it requires a reduced clinical chair time, without compromising the SBS of the bonding interface.

\section{Acknowledgments}

The authors would like to express their appreciation to $3 \mathrm{M}$ UNITEK for providing the APC brackets for this study.

7. Yamamoto A, Yoshida T, Tsubota K, Takamizawa T, Kurokawa H, Miyazaki M. Orthodontic bracket bonding: enamel bond strength vs. time. Am J Orthod. Dentofacial Orthop. 2006 Oct;130(4):435.e1-6.

8. Silta YT, Dunn WJ, Peters CB. Effect of shorter polymerization times when using the latest generation of light-emitting diodes. Am J Orthod Dentofacial Orthop. 2005 Dec;128(6):744-8.

9. Jandt KD, Mills RW, Blackwell GB, Ashworth SH. Depth of cure and compressive strength of dental composites cured with blue light emitting diodes (LEDs). Dent Mater. 2000 Jan;16(1):41-7.

10. Mills RW. Blue light emitting diodes - another method of light curing? Br Dent J. 1995 Mar;178(5):169.

11. Mills RW, Jandt KD, Ashworth SH. Dental composite depth of cure with halogen and blue light emitting diode technology. Br Dent J. 1999 Apr;186(8):388-91.

12. Dunn WJ, Bush AC. A comparison of polymerization by lightemitting diode and halogen-based light-curing units. J Am Dent Assoc. 2002 Mar;133(3):335-41. 
13. Oesterle LJ, Shellhart WC, Belanger GK. The use of bovine enamel in bonding studies. Am J Orthod Dentofacial Orthop. 1998 Nov;114(5):514-9.

14. Bishara SE, Laffoon JF, VonWald L, Warren JJ. Evaluation of nonrinse conditioning solution and a compomer as an alternative method of bonding orthodontic bracket. Angle Orthod. 2001 Dec;71(6):461-5.

15. Artun J, Bergland S. Clinical trials with crystal growth conditioning as an alternative to acid-etch enamel pretreatment. Am J Orthod. 1984 Apr;85(4):333-40.

16. Reynolds IR. A review of direct orthodontic bonding. Br Dent J. 1975 Feb;138(2):171-8.

17. Amra I, Samsodien G, Shaikh A, Lallo R. Xeno III self-etching adhesive in orthodontic bonding: The next generation. Am J Orthod Dentofacial Orthop. 2007 Feb;131(2):160.e11-5.

18. Turk T, Elekdag-Turk S, Isci D. Effects of self-etching primer on shear bond strength of orthodontic brackets at different debond times. Angle Orthod. 2007 Jan;77(1):108-12.

19. Bishara SE, Oonsombat C, Aijlouni R, Denehy G. The effect of saliva contamination on shear bond strength of orthodontic brackets when using a self-etch primer. Angle Orthod. 2002 Nov;72(6):554-7.

20. Davari AR, Yassaei S, Daneshkazemi AR, Yosefi MH. Effect of different types of enamel conditioners on the bond strength of orthodontic brackets. J Contemp Dent Pract. 2007 Jan;8(1):1-7.

21. Fjeld M, Ogaard B. Scanning electron microscopic evaluation of enamel surfaces exposed to 3 orthodontic bonding systems. Am J Orthod Dentofacial Orthop. 2006 Nov;130(5):575-81.
22. Vilchis RJ, Hotta Y, Yamamoto K. Examination of enameladhesive interface with focused ion beam and scanning electron microscopy. Am J Orthod Dentofacial Orthop. 2007 Nov;131(5):646-50.

23. Bishara SE, Ajlouni R, Oonsombat C. Evaluation of a new curing light on the shear bond strength of orthodontic brackets. Angle Orthod. 2003 Aug;73(4):431-5.

24. Cal-Neto JP, Miguel AJ, Zanella E. Effect of a self-etching primer on shear bond strength of adhesive precoated brackets in vivo. Angle Orthod. 2006 Jan;76(1):127-31.

25. Usümez S, Büyükyilmaz T, Karaman AI. Effect of light-emitting diode on bond strength of orthodontic brackets. Angle Orthod. 2004 Apr;74(2):259-63.

26. Mavropoulos A, Staud CB, Kiliaridis S, Krejci I. Light curing time reduction: in vitro evaluation of new intensive light-emitting diode curing units. European J Orthod. 2005 Aug;27(4):408-12.

27. Gronberg K, Rossouw PE, Miller BH, Buschang P. Distance and time effect on shear bond strength of brackets cured with a second-generation Light-emitting Diode Unit. Angle Orthod. 2006 Jul;76(4):682-8.

28. Hajrassie MKA, Khier SE. In-vivo and in-vitro comparison of bond strengths of orthodontic brackets bonded to enamel and debonded at various times. Am J Orthod Dentofacial Orthop. 2007 Mar;131(3):384-90.

29. Anusavice KJ. Phillips Materiais Dentários. $10^{\text {a }}$ ed. Rio de Janeiro: Guanabara-Koogan; 1998. 412 p. 